\title{
Evaluation of the Use of Single Stage Dermal Substitutes in Acute and Chronic Wounds of the Hand
}

\author{
AHMED A MITWALLI, M.Sc.*; AHMED M. EL-BADAWY, M.D.**; AHMED A. HASSAN, M.D.** and \\ NADA A. MAHMOUD, M.D.** \\ The Department of Plastic \& Reconstructive Surgery, Al-Noor Specialist Hospital, Makkah, K.S.A.* and Ain Shams University, \\ Cairo, Egypt**
}

\begin{abstract}
Background: Skin substitutes were developed as an alternative to skin grafts, especially for burn patients. Autologous skin grafting is a painful procedure, and often the extent of damaged skin is too large to be covered by autologous tissue graft in existence of limited donor skin. Our aim of the study was to evaluate the use of dermal substitutes in the treatment of acute and chronic wounds of the hand and to establish an applicable protocol of definitive wound management.
\end{abstract}

Methods: The study included 85 adult patients having acute and chronic wounds of the hand. They were divided into 2 groups Group (A): Fifty-five Patients treated with Single-layer dermal substitutes and STSG applied to the wound bed in a one-stage procedure, group (B): Thirty Patients treated with skin grafting alone. Scar Evaluation was done by Pre-\& post-operative photography. The Vancouver Scar Scale, Histopathological examination at 45 days postoperatively, Range of motion and Patient satisfaction.

Results: Statistical analysis showed significant differences between the two groups.

Conclusion: A true "skin substitute" would act like an autologous skin graft in adhering to the wound bed while providing the physiological and mechanical functions of normal skin. The use of dermal substitutes represents a promising treatment for acute burns and chronic wounds.

Key Words: Biomaterial skin substitutes - STSG - Wounds Hand-Scar assessment scale.

\section{INTRODUCTION}

Recently the emphasis in managing fullthickness defects has shifted from merely reducing morbidity and achieving satisfactory survival to enhancing the long-term prognosis, function and aesthetics of healed wounds [1].

The gold standard for the coverage of these wounds was the use of autologous skin. Autologous skin in the form of split-thickness skin grafts (STSG) is a painful procedure and contains limited thickness of dermal substance in its structure. Therefore, it allow good coverage but with limited functional outcome especially in the full thickness skin defect $[2,3]$.
Advances in molecular biology and tissue engineering has resulted in the development of a number of dermal substitutes with the aim of improving skin quality and, thereby, functional and aesthetic outcomes. This could be attributable to the fact that dermal substitutes serve as support structure for the ingrowth of vessels and autologous fibroblasts. So, filling of the full thickness defect was occurred and the outer coverage only needed $[4,5]$.

Dermal substitute were divided into two broad categories: Biomaterial (acellular) and cellular. Biomaterial skin substitutes are derived from natural or synthetic sources [6].

Whether natural or synthetic, the biomaterial provides an extracellular matrix that allows for ingrowth of surrounding cells. Cellular skin substitutes are distinguished by their origin either xenogeneic (from nonhuman species), autologous (from the patient), or allogenic (from another person) [7].

Halim et al., in 2010 mentioned the dermal substitute as part of the surgeon's reconstructive ladder after local flap if not available as a reconstructive option. However, the role of dermal substitutes in upper limb reconstruction is still evolving with no clear guidance for its use [8].

Another area of concern is cost associated with dermal substitutes. To date there is a single cost analysis study that compared the total costs involved with dermal substitutes reconstructions versus total costs of skin graft only reconstruction for treatment of small-sized burns. Though the total costs among dermal substitutes group culminated higher expenses per patient (2218 Euros), this was not significantly higher than the skin graft only group (1703 Euros) [9].

In this study we evaluate the use of singlestaged acellular dermal substitutes in acute and 
chronic wounds of the hand, as regard the functional and cosmetic outcome and to establish an applicable protocol for definitive wound management.

\section{PATIENTS AND METHODS}

This study has been conducted on eighty five adult co-operative patients with age range from 16 to 65 years old from December 2014 till April 2018. They were presented with either acute or chronic soft tissue defects of the hand. All wounds were clean (not infected) and its surface area was up to $100 \mathrm{~cm}^{2}$.

Exclusion criteria included: Patients with Exposed bones and tendons (without paratenon), Infected wounds, diabetics, or bad general condition.

Patient assessment: Clinical Assessment to the affected hand was done to exclude neurovascular injury and to confirm the Site, size and depth of wound, Movement of the fingers and hand at the level of wrist, metacarpophalangeal and interphalangeal joints. Routine hand X-ray (anteroposterior, oblique lateral, and lateral views) were done to exclude associated fractures. All data had been registered in examination sheet. Then we discussed with the patient the expected results and the importance of postoperative physiotherapy.

Routine serial Pre and post-operative photography were taken at each follow-up visit at 1,3 , 6,12 , and 24 months after surgery.

Preoperative preparation: Preoperative bed preparation by frequent dressing changing, quantitative and qualitative microbiological assay and proper antibiotic use were done.

\section{Surgical procedures:}

Patients were divided into two groups. Group (A) contained of Fifty-five Patients treated with Single-layer dermal substitutes $(1 \mathrm{~mm}$ thickness sheets of dermal matrix Matriderm ${ }^{\circledR}$, MedSkin Solutions Dr. Suwelack AG. after hydration according to the manufacturer's instruction). Then STSG applied to the wound bed in a one-stage procedure. group (B) Thirty Patients treated with STSG alone.

Operation was done under general anesthesia, with tourniquet control. The bed was debrided well reaching a pin-point bleeding in preparation for coverage. Proper hemostasis secured without excessive cauterization to avoid devascularize the bed.

A thin split-thickness graft (0.012-inch thickness) was harvested from the patient's lateral thigh by electric- powered dermatome for final coverage of the wound for both groups with atraumatic tissue handling.

In group A: The dermal substitute was applied to the prepared wound bed after hemostasis and soaked in isotonic saline solution. Autologous meshed skin was grafted over the dermal substitute. The split-thickness skin graft was fixed with staplers or non-absorbable sutures.

In group $B$ : The split-thickness skin graft was fixed alone with staplers or non-absorbable sutures to the prepared bed.

Dermal substitute and STSG was secured in its place to guard against shearing movement. Pressure dressing was done to decrease the seroma formation.

\section{Post-operative:}

First dressing changing was carried on the fifth post-operative day for both groups. Grafted sites were examined at $7^{\text {th }}, 14^{\text {th }}, 21^{\text {th }}$ days after surgery to determine the take rate. The appearance of the grafted area was evaluated and photographed at 1 , $3,6,12$, and 24 months after surgery.

Patient satisfaction were assessed at the end of the follow up period by an interview questionnaire composed of four questions about: Disappearance of symptoms, Quality of the scar, Integration in the career postoperatively and Rating the whole medical care received. We graded our patients" satisfaction on a 5-point scale poor, fair, good, very good and excellent.

Vancouver Scar Scale (VSS) was used for the assessment of the appearance and function of the healed skin. It has four parameters including vascularity, pigmentation, thickness and pliability, giving a range of $0-13$ in the total score.

Histopathological examination was done at 45 days post-operatively to determine Characteristic histological changes in hand wounds treated using dermal substitutes and autografts. It was stressed on blood vessel distribution and organization. Histology was done on ( $\mathrm{n}=10$ patients/group). Formalin-fixed paraffin-embedded tissue sections at a thickness of $3 \mu \mathrm{m}$ were prepared on glass slides. Haemotoxylin and eosin staining (H\&E) was performed using Tissue-Tek Prisma automated stainer (Sakura Finetek Europe, Alphen aan den Rijn, Netherlands). Slides were dewaxed with xylene (Sigma-Aldrich, Gillingham, UK) and rehydrated before being stained with Harris haematoxylin (CellPath, Newtown, UK) for 7 minutes. Excess haematoxylin was removed by washing in a $0.05 \%$ solution of acid alcohol for 10 seconds. Nuclei 
were then blued using Scott's tap water substitute for 1 minute and 15 seconds. Tissue sections were stained with alcoholic eosin (CellPath) for 2 mins. Following staining, slides were dehydrated in $99 \%$ industrial methylated spirit and rinsed in xylene before being mounted using KP coverslipping tape (Klinipath, Olen, Belgium).

\section{Statistical analysis:}

The data were tabulated and statistically analyzed according to Miller MC et al., (1992). Analysis of data was done by IBM computer using SPSS (statistical program for social science version 16) as follows:

- Description of quantitative variables as mean, SD and range.

- Description of qualitative variables as number and percentage.

- Chi-square test was used to compare qualitative variables between groups.

\section{RESULTS}

The study was done over 85 patients, dermal substitutes were used in 55 patients divided into 42 males and 13 females that represented $(76 \%$ and $24 \%$ ) respectively, the other 30 patients without dermal substitutes were used as control group and were divided into 23 males and 7 females that represented (77\% and 23\%) respectively.

The size of the defects in both groups was ranged from $1-80 \mathrm{~cm}^{2}$. The healing was completed in most of cases between 21 to 25 days in both groups. 2ry grafting was needed in 4 patients with dermal substitutes and 7 patients in control group. The defect which required 2ry grafting was minor and can be healed by secondry intention but the patients were operated for an different procedure so it was a chance to accelerate healing by coverage instead of frequent dressing.

The VSS was 4 for 17 patients of dermal substitute group and one patient only of control group. While was 5 for 25 patients of dermal substitute group and 7 patients of control group. The VSS was 6 for 12 patients of dermal substitute group and 13 patients of control group while was 7 for only one patient of dermal substitute group and 8 patients of control group. There is a significant difference between the two groups where $p$-value $=0.00$ Table $(1)$.

Patients satisfaction were variable ranging between (60\%-100\%). As regarding dermal substitute group 25 patients (45\%) were satisfied by $100 \%$ and the same number of patents were satisfied by $80 \%$ and only 5 patients ( $9 \%$ ) were satisfied by
$60 \%$. As regarding the control group 3 patients only $(10 \%)$ were satisfied by $100 \%$, while 21 patients $(70 \%)$ were satisfied by $80 \%$ and 6 patients (20\%) were satisfied by $60 \%$.

Histologic analysis of tissues collected from wounds engrafted with ADM after forty-five days were stained with hematoxylin and eosin. Section of the full-thickness wound treated by skin grafts only showing a hyperkeratotic epidermis with dense fibrotic dermis fibrosis. ADM-engrafted site showed: Regenerated epidermis over fibrotic dermal tissue, effacement of the dermis and subcutis by reparative collagenous connective tissue with elongated fibroblastic/fibrocytic nuclei embedded in collagen in polymer-containing sections, normal basement membrane between epidermis and polymer "dermis"; and granulomatous (foreign body) reaction of epithelioid macrophages and multinucleated giant cells around ADM fibers. ADM in skin sections showed eosinophilic, branching core distinguishing it from surrounding tissue; ADM containing skin with loose fibrovascular penetration and collagen deposition also the epidermis was well differentiated which was similar to normal skin, Rete Peg formation could be seen at the junction between epidermis and dermis, sweat gland like structure also could be seen in the dermis H\&E X400 HPF.

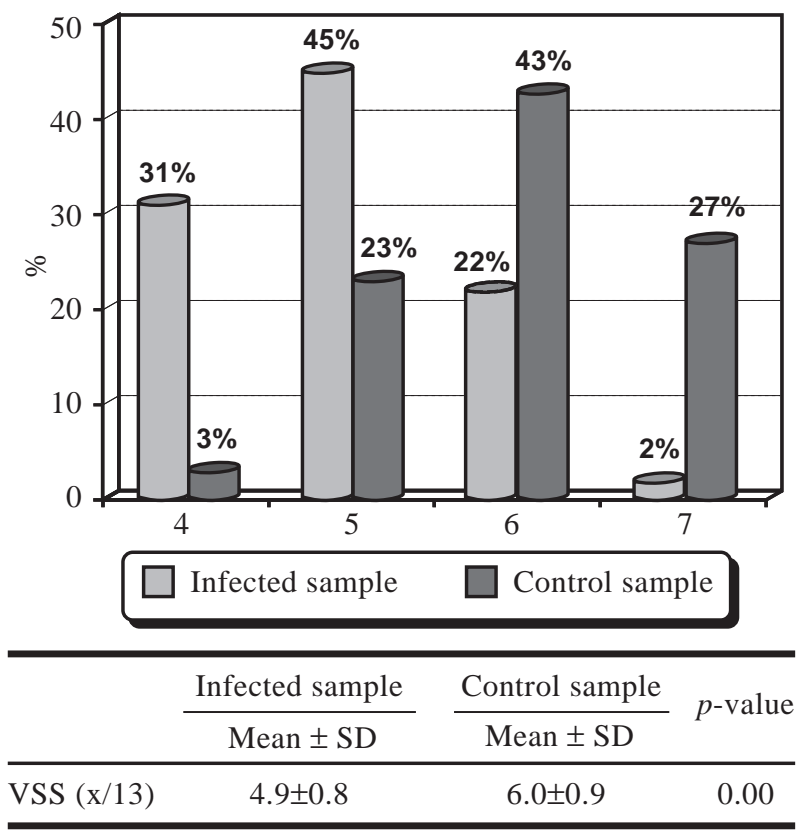

Table (1): The VSS was 4 for 17 patients of dermal substitute group and one patient only of control group. While was 5 for 25 patients of dermal substitute group and 7 patients of control group. The VSS was 6 for 12 patients of dermal substitute group and 13 patients of control group while was 7 for only one patient of dermal substitute group and 8 patients of control group. There is a significant difference between the two groups where $p$-value $=0.00$. 

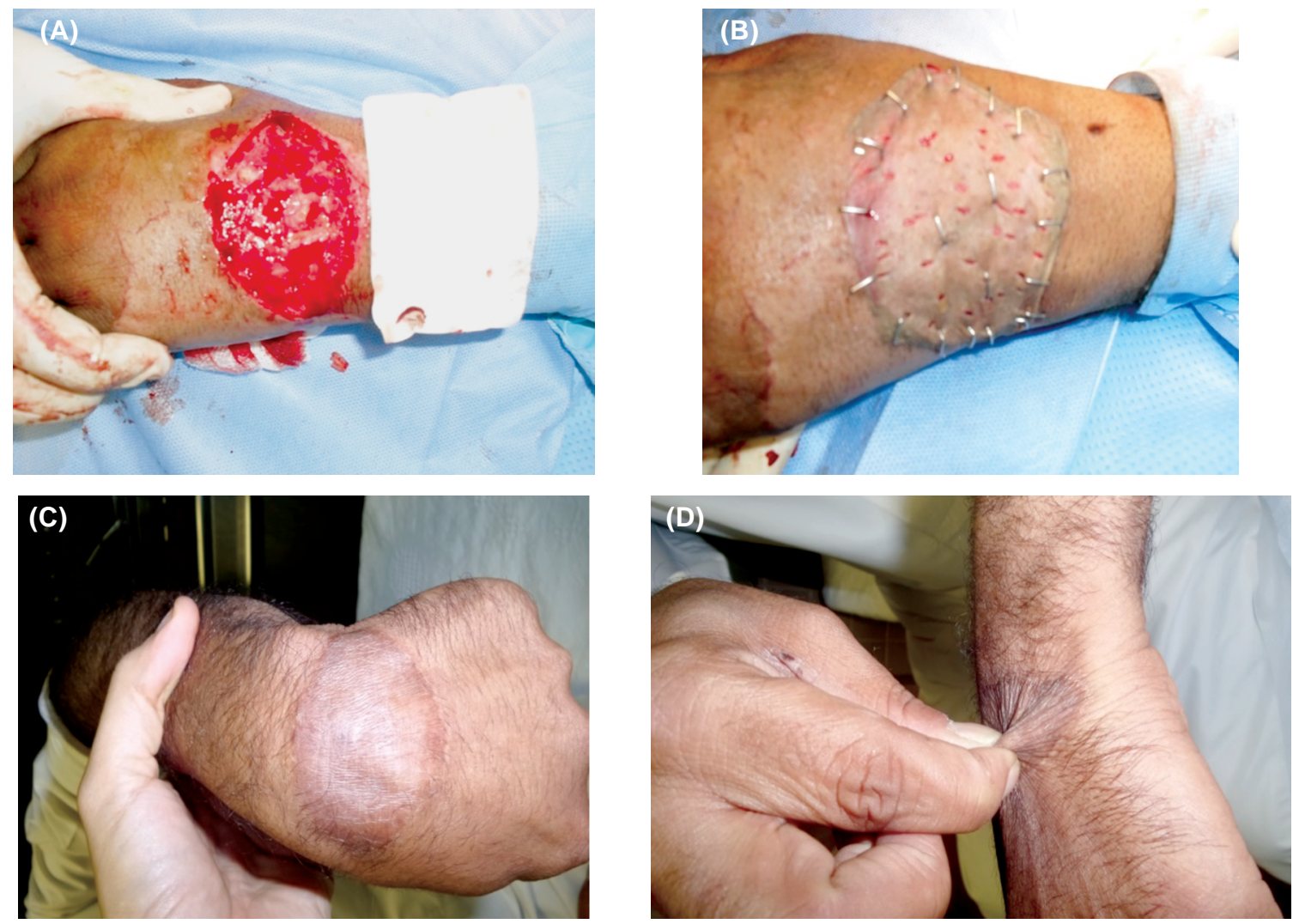

Fig. (1): (A) $9 * 5 \mathrm{~cm}$ defect post debridement full thickness burn dosum of left wrist and hand for reconstruction with STSG and dermal substitute (the white structure on the right side of the picture), (B) $7^{\text {th }}$ post oprative day shows good take, (C,D) 36 months post operatively shows better cosmesis and function.
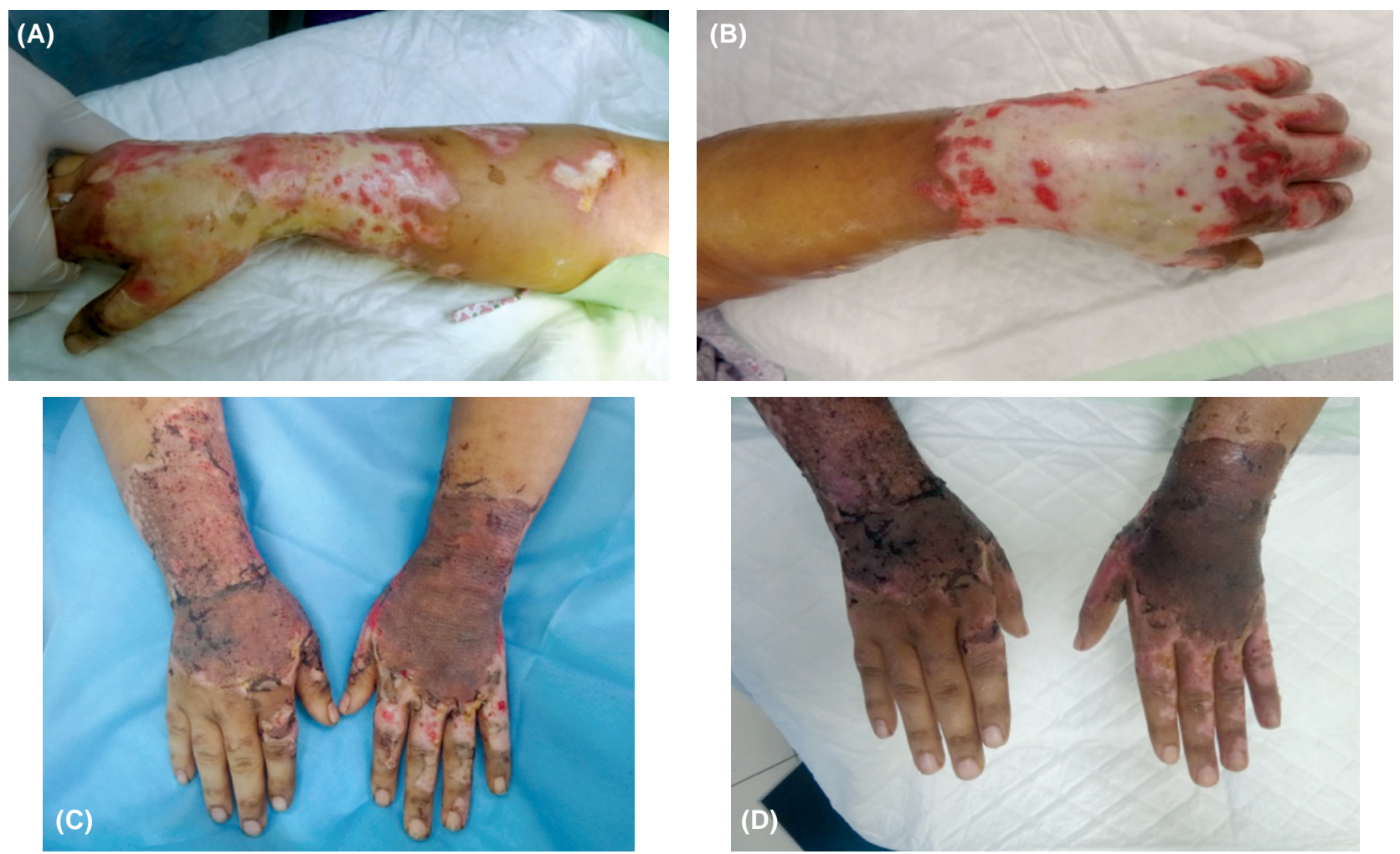

Fig. (2): (A,B) Full thickness burn both hands deeper on the left hand. (B,C) 1 week post reconstruction with dermal substitute and STSG (D) 6 post-op shows good take of the substituted side. 

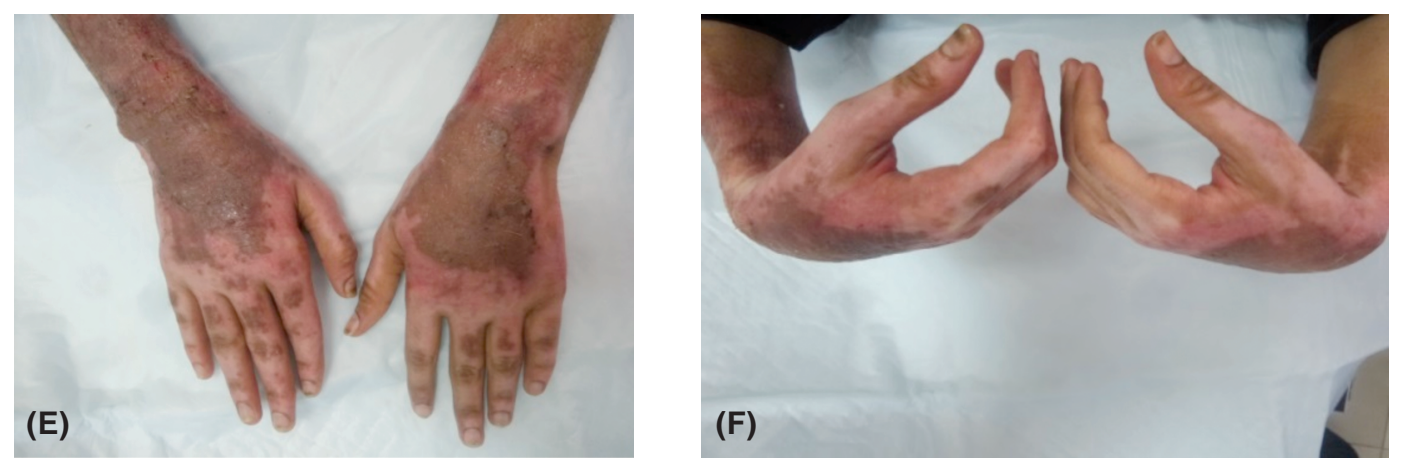

Fig. (2): (E,F) 9 months post operatively shows better cosmesis and function.
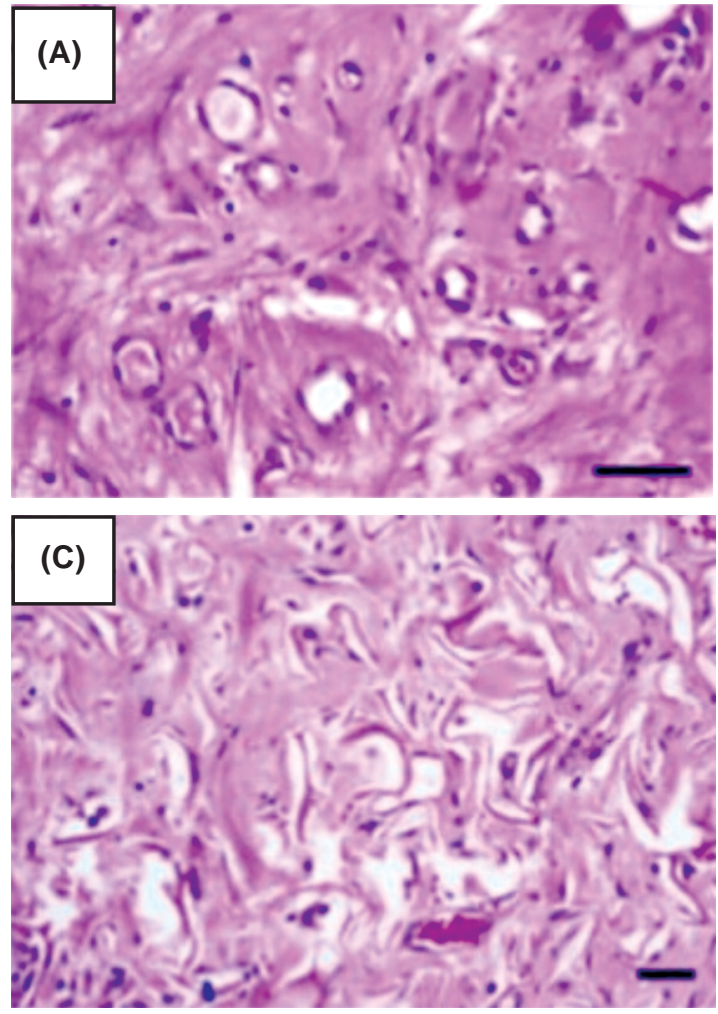

DISCUSSION

With any surgical wound, the goal is rapid healing with a minimal scar that will have a longterm natural contour and provide long lasting tensile strength. Using dermal substitutes in acute and chronic full thickness wounds is thought to lead to better scar elasticity, mechanical stability and appearance. The dermal substitute acts as a support structure giving a three dimentional construct for the neoangiogenesis and invaded by the host fibroblasts [10].

There are many names and types of Acellular Dermal Matrix products. All purporting to be the best formula but all based upon the same principles.

Stressing the importance of dermis in longterm durability and success rates, a number of

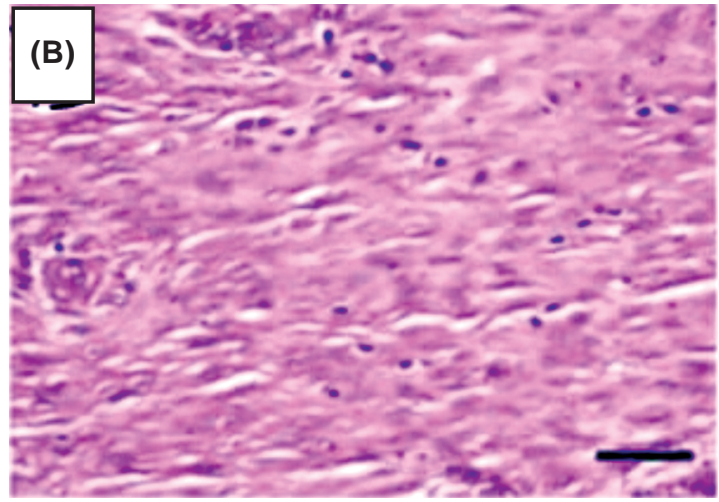

Fig. (3): Histologic analysis of wounds grafted with dermal substitutes after 12 months were stained with hematoxylin and eosin. (A) Higher magnification of normal dermal tissue. (B) Fibrosis of the ungrafted site and dermal substitutes engrafted site and (C) Dermal substitute containing skin with loose fibrovascular penetration and collagen deposition also the epidermis was well differentiated which was similar to normal skin, Rete Peg formation could be seen at the junction between epidermis and dermis, sweat gland like structure also could be seen in the dermis H\&E X400 HPF.

investigators have evaluated a variety of materials as dermal substitutes or scaffolds on which a useful favorable neodermis is formed. However, this bilayer construct requires a two-stage approach, separated by two to four weeks [10].

The newer product, Matriderm (De Suwelack Skin and Health Care AG, Billerbeck, Germany), has demonstrated successful take favorable histologic findings, and improved scarring when applied in acute and reconstructive burn patients, compared to conventional grafting. Matriderm investigators were successful in achieving a one-stage application of dermal substitute and skin graft, and the reconstructive patients had improved elasticity in the short term [11].

Apart from burn surgery there is no clear guidance on the exact indications of dermal substitute 
in upper limb defect for many reasons. First, wounds of the upper extremity have various etiologies and patients often have different co-morbidities. Second, the current available evidence is mostly comprised of non-comparative retrospective case series and individual surgeons' experiences [12].

Therefore, evaluating the effectiveness of dermal substitutes and their long-term functional outcomes in the overall wound healing of the extremity is difficult to interpret [13].

McCauley in 1999, reported results comparable to those with conventional skin grafting in the long-term follow-up of 21 releases in six pediatric patients with major burn injuries. Motykie et al in 2001, reported their results in comparative studies of AlloDerm in the reconstruction of burn scar contractures over joints and on the upper extremity, the lower extremity and hands in the pediatric population. In all these applications, they found that take rates were comparable between the AlloDerm and conventional autograft groups. In addition, the AlloDerm sites required less regrafting and fewer additional reconstructive procedures. Most importantly, a superior range of motion was observed at the AlloDerm sites reaching statistical significance in the lower extremity and hand applications $[\mathbf{1 4 , 1 5}]$.

The thickness of the dermal component is considered an important issue in prevention of wound contraction. Therefore, it is an attractive option to use a thicker sheet of AlloDerm in reconstruction with the potential for a better result than a standard STSG, although this has not been clinically evaluated (Chaudhari et al., 2007). In his study, he reported improved range of motion at sites where AlloDerm was placed for contracture release but stressed the need for compliance and a strict postoperative protocol for success.

Askari et al., in 2006, described their two-stage technique with a cellular dermal matrix (ADM) in nine patients undergoing release of burn contractures of the hand. Following full soft tissue contracture release, including tenolysis and joint capsulotomy where indicated, meshed AlloDerm (0.50$1.05 \mathrm{~mm}$ thick) was applied to the open wound. A thin STSG (0.012 inches) was applied to the ADM 10 to 14 days later. Following both procedures, a vacuum-assisted closure device was incorporated into the immediate postoperative dressing regimen. In long-term follow-up (10 to 25 months), the authors reported excellent maintenance of the improved passive range of motion and web space release achieved at surgery.

This study was carried on 85 patients, dermal substitutes were used in 55 of them with acute and chronic hand defects, the other 30 patients were managed by STSG and were used as control group. Patients satisfaction was $(80 \%-100 \%)$ in $90 \%$ of patients used dermal substitute, however it was $(60 \%-70 \%)$ in $90 \%$ of the control group. Vancouver's scale assessment shows good result in dermal substitute group with a mean of 4.9 , however the mean of the control group was 6 with significant difference between both groups. Histological analysis detected a newly formed dermis in the dermal substitute group rather than the control group. Also, there was good range of motion both active and passive due to application of one stage surgery.

Interestingly, indirect health-related costs such as length of hospital stay and overheads were found to be the most important factors influencing the total cost of treatment. The decision to use dermal substitutes may therefore be a clinical one. Lastly, surgeon's experience and patient preferences should also be considered in the final decision-making process. Nonetheless, dermal skin substitutes have been employed with various degrees of success [19].

After the result mentioned in this study, we applied the use of dermal substitute as a part of our protocol of management in acute and chronic full thickness wound defect in the hand, after clarifying its benefits and the cost difference to our patients especially who are not under the health insurance.

\section{Conclusion:}

The single layered dermal substitutes have demonstrated successful take, favorable histologic findings, and improved scarring when applied in acute and chronic wound, compared to conventional grafting. Matriderm investigators were successful in achieving a one-stage application of dermal substitute and skin graft, and the reconstructive patients had improved elasticity in the short term. A recent 11-year follow-up revealed improved subjective scar assessment measurements and elasticity in the substituted group. Further description of these constructs is beyond the scope of this article, but they have received considerable application in acute burns and reconstructive surgery and are a valuable option for the burn surgeon.

\section{Conflicts of interest:}

None. 


\section{Informed consent:}

Informed consent was obtained from patients before using photographs.

\section{Formatting of funding sources:}

This research did not receive any specific fund.

\section{REFERENCES}

1- Dieckmann C., Renner R., Milkova L., et al.: Regenerative medicine in dermatology: biomaterials, tissue engineering, stem cells, gene transfer and beyond. Exp. Dermatol. Aug., 19 (8): 697-706. PMID: 20545761, 2010.

2- Boyce S.T.: Design principles for composition and performance of cultured skin substitutes. Burns, 27: 523533, 2001.

3- Chern P.L., Baum C.L. and Arpey C.J.: Biologic dressings: Current applications and limitations in dermatologic surgery. Dermatol. Surg. Jun., 35 (6): 891-906. PMID: 19397669, 2009.

4- Buncke G.M., Buntic R.F. and Romeo O.: Pediatric mutilating hand injuries. In: Dellapena D., ed. Hand Clinics. Philadelphia: WB Saunders, 121-131, 2003.

5- Askari M., Fisher C., Weniger F.G., et al.: Anticoagulation therapy in microsurgery: A review. J. Hand. Surg., 31A: 836-846, 2006.

6- Levin L.S. and Cooper E.O.: Clinical use of anticoagulants following replantation. J. Hand. Surg., 33A: 1437-1439, 2008.

7- Halim, Ahmad Sukari, Khoo, Teng Lye, Jumaat, Shah, et al.: Biologic and synthetic skin substitutes: An overview. Indian J. Plast. Surg. Sep., 43 (Suppl): S23-S28. [PubMed: 21321652], 2010.

8- Moiemen N., Yarrow J., Hodgson E., et al.: Long-term clinical and histological analysis of Integra dermal regeneration template. Plast. Reconstr. Surg., 127 (3): 114954. [PubMed: 21088647], 2011.

9- Shirley R., Teare L., Dziewulski P., Frame J., Navsaria H. and Myers S.: A fatal case of toxic shock syndrome associated with skin substitute. Burns. Sep., 36 (6): e968. [PubMed: 20092950], 2010.

10- Ryssel H., Gazyakan E., Germann G. and Ohlbauer M.: The use of MatriDerm in early excision and simultaneous autologous skin grafting in burns: A pilot study. Burns 34: 93-97, 2008.

11- Truong A.T., Kowal-Vern A., Latenser B.A., Wiley D.E. and Walter R.J.: Comparison of dermal substitutes in wound healing utilizing a nude mouse model. J. Burns Wounds, 4: 72-82, 2005.

12- Iorio M.L., Shuck J. and Attinger C.E.: Wound healing in the upper and lower extremities: A systematic review on the use of acellular dermal matrices. Plast. Reconstr. Surg. Nov., 130 (5 Suppl 2): 232S-41S. [PubMed: 23096978], 2012.

13- Jacoby S.M., Bachoura A., Chen N.C., Shin E.K. and Katolik L.I.: One-stage Integra coverage for fingertip injuries. Hand, 1-5. [PubMed: 24426886], 2013.

14- McCauley R.L.: Long term results of acellular dermal matrix (AlloDerm) in pediatric burn reconstruction. J. Burn. Care. Rehabil., 20 (1) (pt 2): S213, 1999.

15- Motykie G.D., Washington W., Sanford A., Herndon N. and Wolf S.E.: Outcomes of AlloDerm grafting in acute severe thermal injuries. J. Burn. Care. Rehabil., 22: S128, 2001.

16- Chaudhari S., Roggy D., Zieger M. and Sood C.R.: Use of Allo- Derm to prevent recontracture following burn scar contracture release. J. Burn. Care. Res., 28 (2) (Suppl): S85, 2007.

17- Askari M., Fisher C., Weniger F.G., et al.: Anticoagulation therapy in microsurgery: A review. J. Hand. Surg., 31 A: 836-846, 2006.

18- Sun Y., Li C. and Wang C.: Study and application of acellular dermal matrix transplantation combined with thin split-thickness autoskin grafting. Chin. J. Plast. Surg., 14: 370-372, 1998.

19- Rizzo M.: The use of Integra in hand and upper extremity surgery. J. Hand. Surg. Am. Mar., 37 (3): 583-6. [PubMed: 22209210], 2012. 